\title{
An Algorithm of Opportunistic Routing Based Network Coding for Satellite Network
}

\author{
Li Yang, Bingjie He, and Huaifeng Shi
}

\begin{abstract}
In order to solve the problem that the throughput decline caused by the time variant of Inter-satellite links in satellite networks, this paper proposes an algorithm of opportunistic routing based on network coding for satellite network, and the maximum average throughput of the satellite network is modeled and solved. It uses network coding on the feedback messages to reduce the redundancy of nodes transmission and guarantee the reachability of neighbor nodes. Simulation results show that the ORCSN algorithm can improve the average throughput of the network, and also can adapts to changes in the loss rates of links.
\end{abstract}

Index Terms-Coded feedback, opportunistic routing, satellite networks, throughput.

\section{INTRODUCTION}

"The Belt and the Road Initiative" which was put forward in March 2015 was regarded as the new opportunity of China's economic development. The great needs of big data transmission, business and entertainment in "The Belt and the Road" program make it significant to make research about data transmission in the satellite network. Compared with traditional routing, using network coding [1] can improve network throughput [2], [3]. Refer to these features such as the satellite network topology dynamically, interstellar link intermittent connection, opportunistic routing [4]-[6] can take advantage of the diversity of the link to provide a better routing strategy. The combination of network coding and opportunistic routing [7], [8] will provide a solution to improve the throughput of satellite network.

Typical opportunistic routing based on network coding have MORE [9] protocol, CCACK [10] protocol, etc. In the paper [9], MORE solves the problem of link collaboration difficult in opportunistic routing through network coding. In the MORE protocol, source node uses the ETX [11] (Expected Transmission Count) of forward nodes to destination node to calculate credit in advance, which determines the node forwarding probability. This way only collects the packets loss rate and calculates credit in the initial state of the link. However, it can not adapt to the dynamic change of satellite network link. CCACK protocol was put forward in the paper [10], which uses the coded feedback approach to reduce the redundancy of network. The upstream

Manuscript received November 26, 2015; revised June 6, 2016. This work was supported by the research on congestion control mechanism of high dynamic and high error satellite communication network (No.61301151, No. 91338104).

The authors are with the Key Laboratory of Communication and Networks, Information Engineering, Dalian University, P. R. China (e-mail: yangli945@126.com, hebingjie321@163.com, shihuaifeng314@126.com). node estimates whether the downstream nodes have received enough packets by the means of coded feedback. But the means of detection link connection state are the same as the methods of MORE. Thus, it can not adapt to the dynamic change of satellite network link either. Besides, in the MORE and CCACK protocol, the rate of source node was heuristic, and no proof of the optimality was provided.

This paper proposes an ORCSN (Opportunistic Routing Based Coding in Satellite Network) algorithm of opportunistic routing based on network coding for satellite network. In this paper, our main ideas are: (1) In order to make the maximum throughput in the network, the source node controls the rate to send each data section. And we use the theory of convex optimization to come up with the optimal solution. (2) The next hop nodes according to return the coded feedback packets of its received packets, which can reduce redundant packets transmission in the network. (3) Each node maintains a table that contains the connections of next-hop nodes in the local. If the link state changes, the node update the table to ensure the reachability with neighbor nodes.

\section{SySTEM MOdEL DESCRIPTION AND THE PROBLEM DEFINITION}

\section{A. Satellite Network Description}

Regarding the satellite network as a directed graph $G(\mathrm{~V}, \mathrm{E})$. $V$ is the vertex set, and indicates the node set in the satellite network. $E$ is the edge set, and indicates the link set in the network. In a satellite network, $S$ is the source node and $D$ is the destination node. The transmission speed of source is $R_{S}$, and the utility function is $U\left(R_{S}\right)$. Since we are using network coding, one important factor that matter is the rate of linearly independent packets that one node has to successfully deliver to next-hop nodes. We use the concept credits to model this factor in the paper [9]. $X_{u v}$ is used to represent the rate of credits transferred from node $u$ to node $v$. Thus, the total rate of credits at node $v$ would be $\sum_{u \in V} X_{u v}$, and these credits will be distributed to the next-hop nodes of $v . R_{u J}$ represents the rate of packets that are sent by node $u$ and are received by any of the nodes in the set $J$. Assume that there are $k$ next-hop nodes for the node $u$, then there are $2^{k}-1$ combinations, and any $J$ belongs to one of the $2^{k}-1$ combinations.

\section{B. The Problem Definition}

The average throughput of the satellite network can be expressed as follows, we want the maximum: 


$$
\text { Maximize } U\left(R_{S}\right)
$$

Subject to:

$$
\begin{gathered}
\sum_{v \in V} X_{v u}-\sum_{v \in V} X_{u v} \leq \begin{cases}-R_{S}, & u=S \\
0, & \text { else }\end{cases} \\
\forall u \in V \backslash D \\
\sum_{v \in J} X_{u v} \leq R_{u J} \quad \forall u \in V \backslash D
\end{gathered}
$$

We assume $U\left(R_{S}\right)$ is the non-decreasing and strictly concave function. Formula (2) shows the total received credits at a node should be equal to the total amount of its sent credits. Formula (3) shows if a packet is received by many nodes, only one node can use this packet to increase its credits.

Proposition 1: It is shows a convex optimization problem from formula (1) to formula (3). In addition, there is no duality gap between the primal problem and its dual problem.

Proof: it can be known by the formula (2) and formula (3) that the constraints are linear, and the intersection of the two half-spaces is a convex set. Simultaneously, the objective function is a concave function, and we want this maximum. Therefore, the problem is a convex optimization problem that can be solved by using dual methods. Besides, because the Slater conditions hold, dual problem and the primal problem do not exist duality gap. So, the optimal solution to the dual problem is the same as the optimal solution to the primal problem.

\section{PROBLEM SOLUTIONS}

\section{A. Structure of Optimal Solution}

Formula (2) multiplied the Lagrange multipliers $\lambda_{u}$, and then the Lagrange function as follows :

$$
L(R, X, \lambda)=U\left(R_{S}\right)-\lambda_{S} R_{S}-\lambda_{u}\left(\sum_{v \in V} X_{v u}-\sum_{v \in V} X_{u v}\right)
$$

Subject to (3).

Making a change on formula (4), it becomes as follows:

$$
L(R, X, \lambda)=U\left(R_{S}\right)-\lambda_{S} R_{S}+\sum_{v}\left(\lambda_{u}-\lambda_{v}\right) X_{u v}
$$

The Lagrange function (5) is a discrete function [12]. This shows that the problem can be solved in a distribution way by using the gradient method.

\section{B. Basic Algorithm}

Source node algorithm: when there have some data to be transmitted in the network, the source node firstly divides the data into batches with equal size, and each batch consists of $k(k>0)$ packets. According to formula (6) as follow, the source node $S$ controls its rate. The index $t$ is the number of batch.

$$
R_{S}(t)=\arg \max \left[U\left(R_{S}\right)-\lambda_{S}(t) R_{S}\right]
$$

Intermediate node algorithm: each intermediate node $u$ assigns the rate of credit for its next-hop according to the formula (7).

$$
\left\{X_{u v}(t)\right\}_{v \in V}=\arg \max \sum_{v \in V}\left(\lambda_{u}(t)-\lambda_{v}(t)\right) X_{u v}
$$

Subject to (3).

Lagrange variables update algorithm: updating the Lagrange variables of each node in recursive method according to formula (8).

$$
\lambda_{u}(t+1)= \begin{cases}\lambda_{u}(t)+\left[\sum_{v \in V} X_{v u}(t)-\sum_{v \in V} X_{u v}(t)\right]+R_{S} & , u=S \\ \lambda_{u}(t)+\left[\sum_{v \in V} X_{v u}(t)-\sum_{v \in V} X_{u v}(t)\right] & , \text { else }\end{cases}
$$

\section{ORCSN ALGORITHM - BASED ON NETWORK CODING}

The basic algorithm which is proposed can make sure the average throughput converges to the optimal solution in this paper. However, it has some shortcomings.

First, the algorithm requires a lot of feedback information. For example, when the source node sends data, the data is divided into batches with equal size, each batch consists of $k$ packets. When sending $k$ packets to the next hop node in $L$, each batch requires $k \times L$ feedback packets. In addition, the loss of satellite link can also increase the difficulty of the feedback information.

Second, it is unable to adapt the dynamic changes of satellite network.

To solve the first problem, this paper intends to compress the feedback information of received packets into a coded packet to reduce the transmission of feedback by using the coded feedback technology.

Aiming at the second problems, this paper intends to maintain a table by using feedback technology on each node. When the link state changes, the node updating this table to make sure the reachability with its neighbor nodes.

\section{A. Coding Feedback Method}

This paper uses zero space to achieve coded feedback. The null space of a matrix is a linear vector space. Therefore, if vector $y$ belongs to the null space of $A$, then $A y^{T}=0$, and $y^{T}$ is the transpose of $y$.

In Fig. 1(a), the vector of each node represent the coefficient of the coded packet, such as the vector $[4,3,1]$ representing the coded packet $" 4 X_{1}+3 X_{2}+X_{3}$ " , and the dotted arrow represents an arbitrary vector of the zero space. In the picture, the node $u$ sends 3 coded packets, and the node $v$ receives 2 of them. The node $v$ can calculate the zero space of the received packets, and then randomly select a vector in the space to the node $u$.

In Fig. 1(b), when the node $u$ receives the zero space vector from its next-hop nodes, it uses the vector to multiply the packets in local cache. If the result is zero, the corresponding position in the table is labeled. It is means that the packet has a large probability to be received by node $v$. Otherwise, the node $u$ knew that the packet has not been successfully received by the node $v$. We can use a hash table 
implementation it, and the probability of wrongful is about $10^{-10}[11]$.

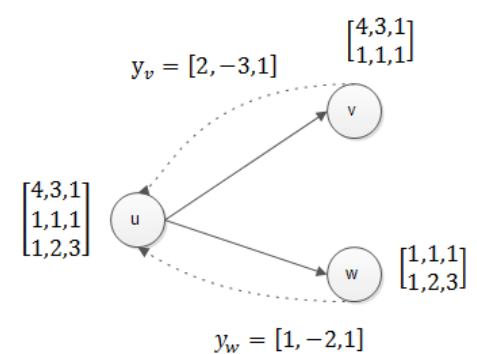

Fig. 1. (a). A case of zero space vector by coded feedback.

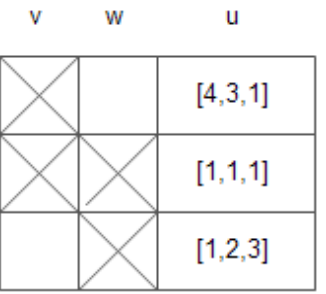

Fig. 1. (b). Built a table by feedback vector.

\section{B. Maintain the Link State Table}

In our paper, each node maintains a link-state table locally by coded feedback. Link state include the next-hop nodes of one node and the metric of their links. The metric is the Lagrange variables difference of the node and its next-hop nodes, which is a routing criterion of this algorithm. This will be described in detail in next part.

TABLE I: THE TABLE's FORMAT OF LINK STATE

\begin{tabular}{lllll}
\hline \hline $\begin{array}{l}\text { Next hop } \\
\text { node }\end{array}$ & Metric & \multicolumn{2}{l}{$\begin{array}{l}\text { Connected } \\
\text { state }\end{array}$} & \multicolumn{2}{l}{$\begin{array}{l}\text { Time } \\
\text { feedback }\end{array}$} \\
\hline$\cdots$ & $\cdots$ & & $\cdots$ & $\cdots$ \\
\hline \hline
\end{tabular}

If one node is connected to its next-hop nodes, the connected state is 1 in the table of link state in Table I. And when the link is interrupted, the connected state is 0 .

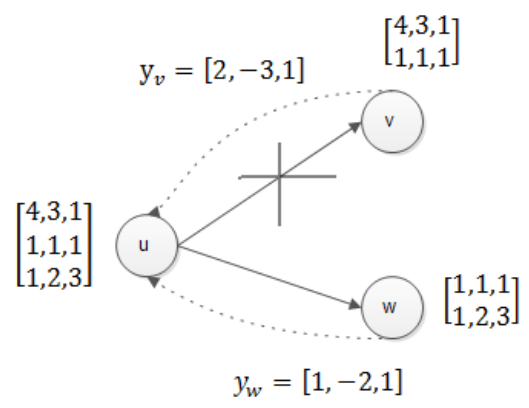

Fig. 2. A case of the link is interrupted.

\begin{tabular}{cccc}
\multicolumn{2}{l}{ TABLE II: UPDATE LINK STATE TABLE BASED ON LINK CHANGE } \\
\hline \hline $\begin{array}{l}\text { Next hop } \\
\text { node }\end{array}$ & Metric & $\begin{array}{l}\text { Connected } \\
\text { state }\end{array}$ & $\begin{array}{l}\text { Time of receive } \\
\text { feedback packet }\end{array}$ \\
\hline$v$ & 4 & 0 & $T 1$ \\
$w$ & 7 & 1 & $T 2$
\end{tabular}

At the same time, a timer is set on each node. We can know the time $T$ that one node sends the packets and receives the feedback from its next-hop nodes. If there is no feedback packets be received by the sending nodes during $2 T$, which means that the link is interrupted, and the connected state in the table is updated to 0. As shown in Fig. 2 and Table II. When the table of link state is changed, the node resends the probe packets finding new neighbors.

\section{Intermediate Node Coded Feedback Algorithm}

In this paper, each of the intermediate nodes need to allocate the rate of credits for its next-hop nodes. Thus, the intermediate nodes need to deal with the problem, as follow: (1) the intermediate nodes determine the next-hop node set $J$; (2) when the intermediate node sends the current packets, it allocates the rate of credits for some nodes in set $J$.

When the intermediate node deals with the first question, it needs to find the optimal next-hop nodes set. According to the formula (3) and formula (7) we can know that the objective function (7) is a linear function of $X_{u v}$. In order to satisfy the constraint (3), the node $u$ needs to store the value of $\lambda_{u}-\lambda_{v}$. The node which has a much bigger difference is that it preferentially gets the rate of credits. Once a node gets the rate of credits, this node belongs to set $J$ during the process of sending data. The set $J$ is the best next-hop nodes for this intermediate node.

For the second question, the formula (3) shows that when the next-hop node $v$ receives a coded packet and no other nodes who has a more bigger difference receive the packet, the node $v$ can use this packet to increase its rate of credits. If the intermediate node detects that its rate of credits equal to the value received by its next-hop nodes, the node stops sending data. In this paper, we use coded feedback to achieve the process.

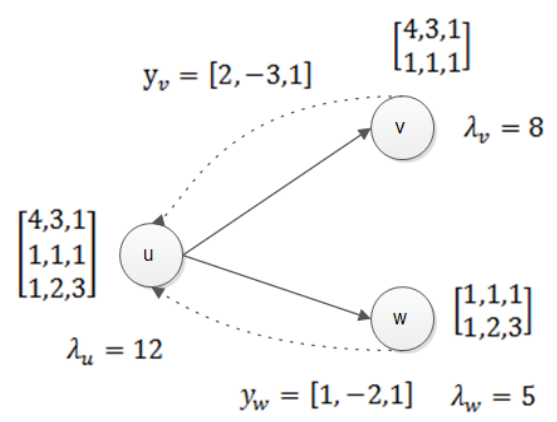

Fig. 3. A example of the credit rate allocation.

As shown in Fig. 3, the node $u$ has three coded packets, this means that the node $u$ has three credits will send to its next-hop nodes. The node $v$ received two packets, and the node $w$ also received two packets. Through the zero space vector of node $v$ and $w$, the node $w$ is allocated 2 credits and the node $v$ is allocated 1 credit because the node $w$ has a much bigger Lagrange variable difference.

\section{Confirmation the Data}

When the destination node receives the coded packets, the node adds them to the coded matrix of the current data batch. Once the destination node successfully decoded the original data, it is immediately send an ACK packet to the source node.

In our algorithm, the priority of ACK packet is higher than other packets. In order to ensure the ACK packet could be delivered fastest, the algorithm use the nodes which have a 
bigger difference to deliver the ACK packet.

When the source node receives an ACK packet of the current data batch, it updates the cache and starts to send the next data batch. And once the other intermediate node got the ACK packet or another data batch's coded packets, the intermediate node would stop sending the current data.

\section{The Simulation And Analysis of Performance}

\section{A. The Settings of Simulation Parameters}

In this paper, we are using a likeness of Iridium as the simulation network topology. The network parameters are shown in Table III. The adjacent satellites on same orbital plane is always connected in the simulation network. And there are 12 links between the satellites on different orbital planes.

TABLE III: THE SiMULATION NETWORK PARAMETERS

\begin{tabular}{ll}
\hline \hline The parameter name & The parameter value \\
\hline Altitude & $1280 \mathrm{~km}$ \\
Orbital plane number & 3 \\
Satellite in orbit number & 6 \\
Orbital inclination & $86.4^{\circ}$ \\
\hline \hline
\end{tabular}

As shown in Fig. 4, we are using STK software to set up the satellite model and calculate all links connection during 24 hours. The symbol S1, S2, S3 represent the orbital plane of this network topology. S101-S106 represent the six satellites on S1, and the symbol S101_201 is the link between the satellite S101 and S201. There are 12 different colors which represent the links between different orbital plane in Fig. 4. The connective times of links is described by horizontal line. We can calculate the cycle of the model is 112 minutes, and the model has 24 snapshots in a cycle.

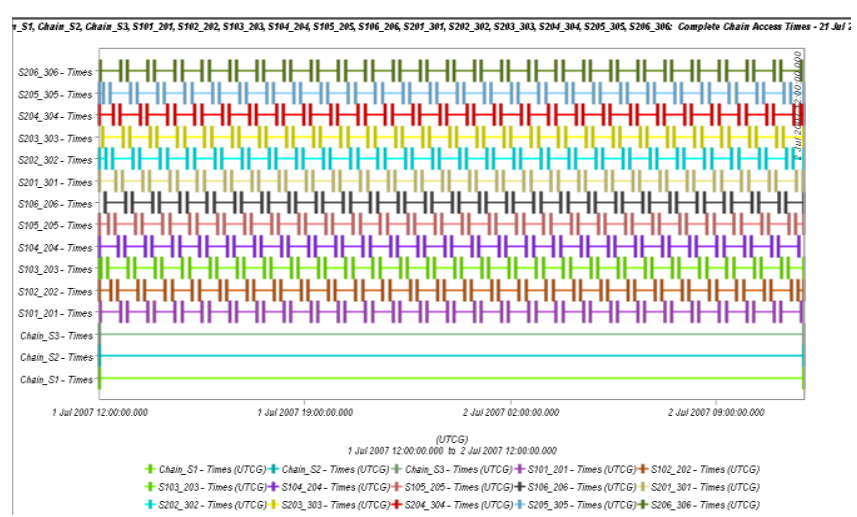

Fig. 4. Link connection during 24 hours.

The Fig. 5 is the satellite network topology at a moment. In this topology, $S$ is the source node and $D$ is the destination node. The finite field of network coding is $G F\left(2^{8}\right)$. The data packet's size is 512 byte, and the utility function $U\left(R_{S}\right)$ is $\log \left(1+R_{S}\right)[13]$.

\section{B. Maintain the Link State Table Results of Simulation}

In Fig. 6, it compares the throughput with different link failure rate for different algorithm. Link failure rate is the percentage of packets which are not able to reach on this link. Throughput computes formula as follows:
Average throughput $=\frac{\text { end to end packet total number }}{\text { total elapsed time }}$

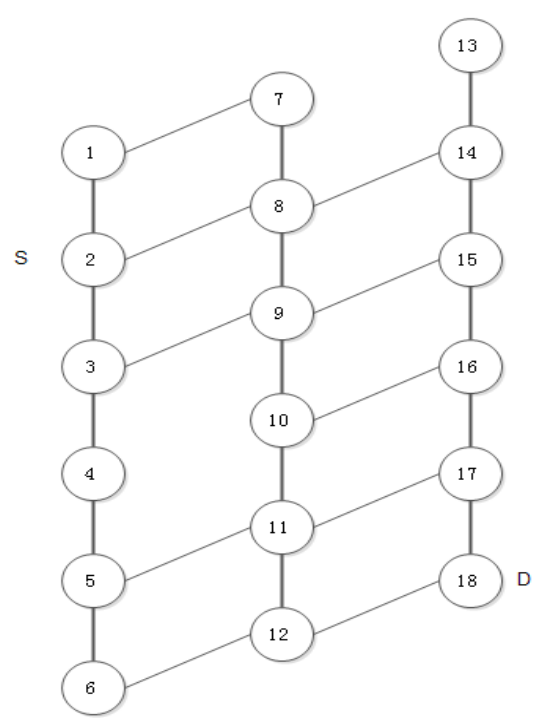

Fig. 5. The satellite network topology.

As it can be seen from the Fig. 6, the throughput of the different algorithms will decrease with the increase of link failure. The reason is that the increase of ink failure rate will lead to a package need transfer more times in the network. This means that the transport time becomes longer than before. But the ORSCN algorithm improves the throughput from $25 \%$ to $40 \%$ over both MORE and CCACK, and improve $90 \%$ over the Dijstra algorithm. In addition, the rate of source node was adjusted by maximizing throughput in ORSCN algorithm, while the rate was based on a heuristic way in MORE and CCACK. Thus, the dropping speed of throughput in our algorithm is lower than others with the increase of link failure.

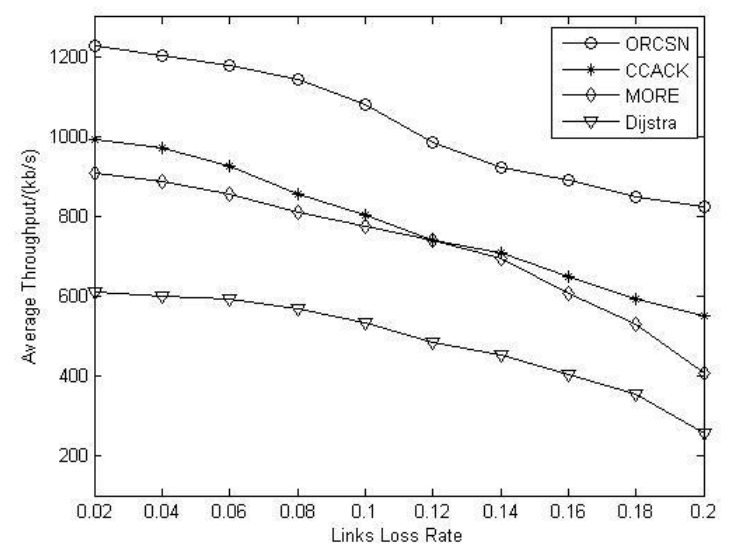

Fig. 6. The throughput about different link failure.

In Fig. 7, it shows the changes of throughput in a cycle in this satellite network topology. As it can be seen from the graph, the throughput of ORSCN algorithm tend to be stable in a cycle. This means that ORSCN algorithm can adapt to the dynamic change of satellite network topology. While the throughput of CCACK and MORE have varying degrees of decline in a cycle. This is because the CCACK and MORE detect the links at the beginning of simulation, then they default the network topology and it will no longer changed. 
Due to the changing of link in the satellite network, using CCACK and MORE can causes the decline of throughput when the topology changed. It makes throughput loss less than MORE algorithms by adopting the method of coded feedback in CCACK. Only when the shortest path is connected, the Dijstra algorithm can be used to transmit data packets.

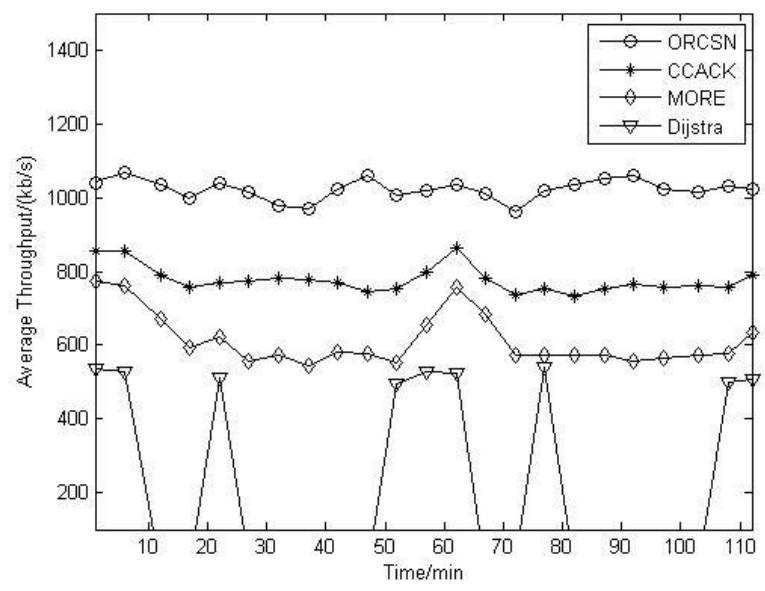

Fig. 7. Throughput simulation within a period.

\section{CONCLUSION}

Aiming at the problem that the decline of the throughput is caused by the time variability of satellite network, the paper proposes an opportunity routing algorithm based on network coding for satellite network. The simulation results show that the ORCSN algorithm is better than MORE and CCACK, which can improve the throughput from $25 \%$ to $40 \%$. The algorithm can adapt to the characteristics of links in satellite network and adapt to the changes of link failure. The future works are combining the intra-session network coding and inter-session network coding to improve the throughput in the satellite network, and designing a reasonable algorithm which can ensure the effectiveness of data transmission when there are multiple data streams in network.

\section{REFERENCES}

[1] S.-Y. R. Li, R. W. Yeung, and N. Cai, "Linear network coding," IEEE Transaction Information Theory, vol. 49, no. 2, pp. 371-381, 2003.

[2] W. Xiaoyan and L. Jie, "Network coding aware cooperative MAC protocol for wireless Ad Hoc networks," IEEE Transactions on Parallel and Distributed Systems, vol. 22, 2013.

[3] N. Shufen, W. Caifen, Z. Yulei et al., "Data integrity verification scheme for multi-source network coding," Computer Engineering, vol. 41, no. 3, pp. 21-25, 2015.

[4] R. Cioban, R. Marin, C. Dobre et al., "Interest-awareness in data dissemination for opportunistic networks," Ad Hoc Networks, vol. 25, pp. 330-345, 2015.
[5] F. Wu, T. Chen, S. Zhong et al., "A game-theroretic approach to stimulate cooperation for probabilistic routing in opportunistic networks," IEEE Trans on Wireless Communications, vol. 12, no. 4, pp. 1573-1583, 2013.

[6] R. Ciobanu, R. Marin, C. Dobre et al., "Interest-awareness in data dissemination for opportunistic networks," Ad Hoc Networks, vol. 25 pp. 330-345, 2015

[7] W. P. Wang, X. Z. Chen, M. M. Lu, and J. X. Wang, "Network coding based opportunistic routing using cumulative coding coefficient feedback acknowledgments," Ruan Jian Xue Bao/Journal of Software, vol. 25, no. 7, pp. 1541-1556, 2014.

[8] D. Ferreira and R. A. Costa, "Real-time network coding for live streaming in hyper-dense WiFi spaces," IEEE Journal on Seletcted Areas in Communications, vol. 32, no. 4, pp. 773-781, April 2014.

[9] S. Chachulski, M. Jennings, S. Katti et al., "Trading structure for randomness in wireless opportunistic routing," ACM SIGCOMM Computer Communication Review, vol. 37, no. 4, pp. 169-180, 2007.

[10] D. Koutsonikolas, C. C. Wang, and Y. Hu, "CCACK: Efficient network coding based opportunistic routing through cummulative coded acknowledgment," in Proc. IEEE INFOCOM Conference on Computer Communications, Purdue Univ., West Lafayette, USA, Mar 2010, pp. 1-9.

[11] D. S. de Couto, D. Aguayo, J. Bicket, and R. Morris, "A hige-throughput path metric for multi-hop wireless routing," Wireless Networks, vol. 11, no. 4, pp. 419-434, 2005.

[12] T. Bonald and L. Massoulie, "Impact of fairness on Internet performance," in Proc. ACM Joint Int'l Conf. on Measurement and Modeling of Computer System(Sigmetrics), Cambridge, MA, June 2001.

[13] S. Boyd and L. Vandenberghe, Convex Optimization, Cambridge University Press, 2004.

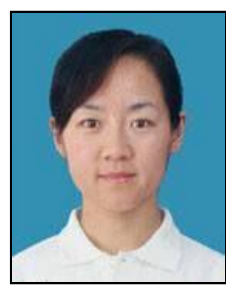

Li Yang was born in January 1982 in Heilongjiang Province. She received his Ph.D. degree in Nanjing University of Science and Technology. Her researches include the theory and method of wireless network protocol.

In 2009, she joined the Dalian University and now she is a professor of Dalian University. Her research has won the country's scientific and technological progress in P.R China.

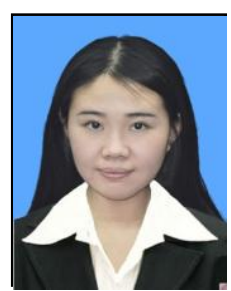

Bingjie He was born on July 29, 1990 in Shanxi Province. She received his master's degree and bachelor's degree in Dalian University. In 2013, She engaged in scientific research in communications and networking key laboratory. Her main research direction is wireless network routing technology.

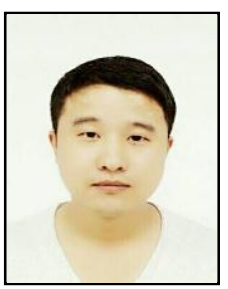

Huaifeng Shi was born on March 1, 1989 in Jiangsu Province. He received his master's degree and bachelor's degree from Nanjing University of Science and Technology in 2014 and 2011, respectively. After that, he joined the Dalian University as a teaching assistant, and engaged in scientific research in communications and networking key laboratory in April 2014. His main research direction is wireless communication and network technology. 